\title{
Life at the periphery of the Local Group: the kinematics of the Tucana dwarf galaxy ${ }^{\star}$
}

\author{
F. Fraternali ${ }^{1}$, E. Tolstoy ${ }^{2}$, M. J. Irwin ${ }^{3}$, and A. A. Cole ${ }^{4}$ \\ 1 Department of Astronomy, University of Bologna, via Ranzani 1, 40127 Bologna, Italy \\ e-mail: filippo.fraternali@unibo.it \\ 2 Kapteyn Astronomical Institute, University of Groningen, Postbus 800, 9700 AV Groningen, Netherlands \\ e-mail: etolstoy@astro.rug.nl \\ 3 Institute of Astronomy, University of Cambridge, Madingley Road, Cambridge CB3 OHA, UK \\ e-mail: mike@cam.ast.ac.uk \\ 4 School of Mathematics \& Physics University of Tasmania, Private Bag 37 Hobart, 7001 Tasmania, Australia \\ e-mail: andrew.cole@utas.edu.au
}

Received 19 August 2008 / Accepted 19 March 2009

\begin{abstract}
Aims. Dwarf spheroidal galaxies in the Local Group are usually located close to the Milky Way or M31. Currently, there are two clear exceptions to this rule, and the Tucana dwarf galaxy is the most distant at almost $1 \mathrm{Mpc}$ from the Milky Way. Our aim is to learn more about the nature of Tucana by measuring its radial velocity and internal kinematics.

Methods. Using the VLT/FORS2 spectrograph in multi-object mode we were able to measure the velocities of 23 individual red giant branch stars in and around Tucana using the Ca II triplet absorption lines. From this sample 17 reliable members have been identified. Results. We measured the systemic velocity and dispersion of Tucana to be $v_{\text {hel }}=+194.0 \pm 4.3 \mathrm{~km} \mathrm{~s}^{-1}$ and $\sigma_{\text {l.o.s. }}=15.8_{-3.1}^{+4.1} \mathrm{~km} \mathrm{~s}{ }^{-1}$, respectively. These measures are obtained after removing the signature of rotation using a linear gradient of $6.5 \times R / R_{\text {core }} \pm 2.9 \mathrm{~km} \mathrm{~s}{ }^{-1}$ which corresponds to a rotation of $\approx 16 \mathrm{~km} \mathrm{~s}^{-1}$ at the reliable limit of our data. Our systemic velocity corresponds to a receding velocity from the barycentre of the Local Group of $v_{\mathrm{LG}}=+73.3 \mathrm{~km} \mathrm{~s}^{-1}$. We also determined the mean metallicity of Tucana to be $[\mathrm{Fe} / \mathrm{H}]=-1.95 \pm 0.15$ with a dispersion of $0.32 \pm 0.06 \mathrm{dex}$.

Conclusions. Our study firmly excludes any obvious association of Tucana with the HI emission in the vicinity and shows that Tucana is a genuine dwarf spheroidal with low metallicity stars, no gaseous ISM and no recent star formation. The present location and relatively high recession velocity are consistent with Tucana having been an isolated Local Group galaxy for the majority of its existence.
\end{abstract}

Key words. galaxies: individual: Tucana dwarf - galaxies: structure - galaxies: kinematics and dynamics - galaxies: stellar content

\section{Introduction}

Due to their faintness, dwarf spheroidal galaxies have only been, at the moment, observed and studied in detail in the Local Group. They are characterized by complex past star formation histories, the lack of present-day gas, and thus a lack of ongoing star formation (e.g., Mateo 1998). Most of them tend to lie very close to the giant spirals [the Milky Way and M31], and interactions with these could be the reason for the lack of gas. There are two currently known exceptions: Cetus, located at $775 \mathrm{kpc}$ from the Milky Way and $680 \mathrm{kpc}$ from M 31 (Whiting et al. 1999; Lewis et al. 2007), and Tucana, which lies some $900 \mathrm{kpc}$ from the Milky Way and $1350 \mathrm{kpc}$ from M 31 (Lavery \& Mighell 1992; Saviane et al. 1996). A third galaxy, And XVIII may fall in the same category (McConnachie et al. 2008).

The association of Tucana with the Local Group is relatively recent (Lavery \& Mighell 1992). Color-magnitude diagrams obtained with CCD photometry (Saviane et al. 1996; Castellani et al. 1996) show no evidence of recent star formation and suggest that Tucana has been completely dormant for the last 8-10 Gyr. There is also clear evidence of an ancient stellar

* Based on FORS1 and FORS2 observations collected at the European Southern Observatory, proposals 63.N-0560 and 69.B-0305. population, as demonstrated by an extended horizontal branch (Tolstoy et al. 2000; Holtzman et al. 2006) and RR Lyrae stars (Bernard et al. 2008). The average metallicity, derived by comparing the color-magnitude diagram (CMD) with globular clusters, has been estimated to be between $[\mathrm{Fe} / \mathrm{H}]=-1.6$ and -1.8 (Saviane et al. 1996; Castellani et al. 1996). These physical properties suggest Tucana is a typical dwarf spheroidal (dSph) galaxy but with the peculiarity of being located at a large distance from the luminous members of the Local Group, and $\approx 1175 \mathrm{kpc}$ from the Local Group barycentre, see Table 1.

A search for neutral gas (HI at 21-cm) associated with Tucana has been carried out by Oosterloo et al. (1996) using the Australian Telescope Compact Array (ATCA). They found an upper limit on the $\mathrm{HI}$ mass associated with Tucana of $M_{\mathrm{HI}} / L_{\mathrm{B}}$ of $\approx 10^{-2}$ which is consistent with values for other dSphs (Mateo 1998; Young 2000). In the same data, an H I cloud was found at a projected distance of $15^{\prime}$ from Tucana (3.4 kpc at its distance) with mean heliocentric velocity of $130 \mathrm{~km} \mathrm{~s}^{-1}$. This velocity is close to that of the Magellanic Stream cloud complexes in that region (Putman et al. 2003) and Oosterloo et al. (1996) suggested this as the most probable association of the cloud. On the other hand, other authors have argued in favour of an association with Tucana (e.g. Blitz \& Robishaw 2000; Bouchard et al. 2006). 
Table 1. Tucana dwarf physical parameters.

\begin{tabular}{lcc}
\hline \hline Parameter & Tucana dwarf & Ref. \\
\hline Type & dSph & - \\
RA, Dec $(\mathrm{J} 2000)$ & $224149.6,-642511$ & $(1)$ \\
$l, b\left(^{\circ}\right)$ & $322.9,-47.4$ & - \\
Distance $(\mathrm{kpc})$ & 880 & $(2)$ \\
$L_{\mathrm{V}}\left(10^{5} L_{\odot}\right)$ & 5.5 & $(3)$ \\
$v_{\text {sys }}$ & 194.0 & $(4)$ \\
$R_{\text {core }}\left({ }^{\prime \prime}\right)$ & 42 & $(3)$ \\
$R_{\text {tidal }}\left({ }^{\prime \prime}\right)$ & 222 & $(3)$ \\
PA $\left(^{\circ}\right)$ & 97 & $(3)$ \\
$e$ & 0.48 & $(3)$ \\
$V_{\mathrm{HB}}$ & 25.4 & $(4)$ \\
Conversion $1^{\prime \prime} / \mathrm{pc}$ & 4.3 & - \\
\hline
\end{tabular}

(1) Lavery \& Mighell (1992); (2) Castellani et al. (1996); Saviane et al. (1996); (3) Mateo (1998); (4) this work.

In this paper we present results from FORS2 multi-object spectroscopy of a sample of red giant branch (RGB) stars in and around Tucana (see Fig. 1). Using these data we determine an accurate optical radial velocity of the galaxy (Sect. 3.1) by fitting $\mathrm{Ca}$ II triplet absorption lines. We also study the internal kinematics of Tucana (Sect. 3.2) and estimate the metallicity of the RGB stars using Ca II triplet equivalent widths (Sect. 3.4). We then discuss the possible association of Tucana with the $\mathrm{H}$ I cloud and its unusual location within the Local Group (Sect. 4).

\section{Observations and data reduction}

The observations of Tucana were obtained in service mode using the UT4/FORS2 spectrograph at the VLT over 3 nights in August 2002 in the multi-object (MXU) mode (see Table 2). We used the GRIS_1028z grism and the OG590 filter in order to cover the spectral region of the Ca II triplet absorption lines. A total of 45 slits were placed on individual stars over a spatial region of about $8^{\prime}(\approx 2 \mathrm{kpc})$ centred on Tucana. Out of the 45 slits, 30 were located on positions of stars selected to lie on the RGB in the CMD (see Fig. 2), and the remaining targets were randomly selected to fill otherwise empty slits. The spatial size of each slit is $1.2^{\prime \prime} \times 8^{\prime \prime}$, the dispersion scale is $0.86 \AA \operatorname{pixel}^{-1}\left(\approx 30 \mathrm{~km} \mathrm{~s}^{-1}\right)$ leading to a velocity resolution (from the dispersion of Gaussianlike unresolved sky lines) of about $50 \mathrm{~km} \mathrm{~s}^{-1}$.

Standard data reduction steps were carried out with IRAF (Image Reduction and Analysis Facility) and with our own IDL routines. Observations from the same night were combined together in order to remove cosmic rays. In total 12 exposures of $1560 \mathrm{~s}$ were made for a total integration time of $5.2 \mathrm{~h}$. We first corrected for distortions of the focal plane by fitting a 3rd order polynomial function to the position of the objects. Since the objects are much fainter than the sky lines, we performed the wavelength calibration using the sky lines themselves. In order to determine the wavelength solutions, we fitted polynomial functions of an order that depends on the number of identified lines, but was typically of order 3 with the maximum a 4th order polynomial. The accuracy of the wavelength solution was tested by fitting Gaussian functions to sky lines of well known wavelengths around the Ca II triplet region (8450-8700 ̊), after the wavelength calibration had been applied. The resulting errors are typically $\approx 5 \mathrm{~km} \mathrm{~s}^{-1}$. Looking at the through-slit images taken before and after each spectroscopic sequence all the objects with successfully extracted spectra were well centred in the slit and we did not need to make additional corrections to the radial velocities for slit mis-centering (e.g., Tolstoy \& Irwin 2000).

Sky subtraction was carried out by fitting a 1 st or 2 nd order polynomial function to the object-free regions along the spatial direction after correcting for the bending of the lines along the spatial axis. In order to improve the extraction, the 2D spectra were then smoothed to a velocity resolution of about $60 \mathrm{~km} \mathrm{~s}^{-1}$ dispersion. After sky subtraction, the spectra of the objects were extracted along the spatial axis. Gaussian functions were fitted to the data (after binning along the dispersion axis) and the object spectra extracted by summing a spatial region typically within $\pm 2 \sigma$ from the central positions. Although the slits have been straightened at this stage, there may be a residual displacement (typically \pm 1 pixel) of the objects with respect to their average positions therefore the objects have been further traced using a polynomial function.

Once the spectra have been extracted, the Ca II triplet absorption lines were used to determine the radial velocities of the stars by fitting a triple-Gaussian function template with fixed relative distances between the three Gaussians. The relative intensity ratios of the Ca II lines were also fixed by calibrating them on the globular cluster M 15. Finally, we assumed an equal velocity dispersion for the three lines. With these assumptions the free parameters of the Ca II fitting are: 1) the position along the dispersion axis (radial velocity); 2) the global intensity scaling and the 3) dispersion of the lines. Two more parameters are used to fit the underlying continuum, which is assumed to be linear in the region of interest.

\section{Results}

Table 3 shows the stars for which we obtained an acceptable fit for the Ca II triplet lines (signal-to-noise per $\AA$ > 14). The spatial locations of these stars are shown in Fig. 1, where $N$ refers to the slit number which is also plotted in Fig. 1. The quality of these fits was also checked with the cross-correlation of the Ca II triplet-Gaussian model with the full spectrum. All the stars included in Table 3 show a clear peak in the cross-correlation. As an example of the quality of the data, Fig. 3 shows the spectrum obtained for star \#4 in Table 3.

\subsection{Systemic velocity and velocity distribution}

The stars in Table 3 are sorted by increasing projected geometrical radial distance from the centre of Tucana. The final sample of most probable members excludes: the 3 obvious likely non-member stars with velocities below $150 \mathrm{~km} \mathrm{~s}^{-1}$; star \#10, which is clearly a double star in the imaging data and also has slit mis-centering problems; star \#21, which does not have any accurate photometric information; and the star \#22, which lies outside the tidal radius. For the purposes of analysis we exclude the above 6 stars, but we note that \#10,\#21 and \#22 are still potential Tucana members. If \#21 and \#22 are included in the analysis there is no significant change to any of the results.

Figure 4 shows the distribution with projected geometric radius (elliptical radius) for all 23 stars observed (left hand panel), and a histogram with a Gaussian model fit determined from a maximum likelihood analysis of the distribution of the radial velocities of the 17 likely members (right hand panel). The 2D maximum likelihood fit together with the marginalised $1 \mathrm{D}$ velocity dispersion is shown in Fig. 5. We find the systemic heliocentric velocity to be $v_{\text {helio }}=+193.0 \pm 4.9 \mathrm{~km} \mathrm{~s}^{-1}$ $\left(V_{\mathrm{GC}}=+97.9 \mathrm{~km} \mathrm{~s}^{-1}\right)$ with a dispersion of $\sigma=17.4_{-3.5}^{+4.5} \mathrm{~km} \mathrm{~s}^{-1}$, 


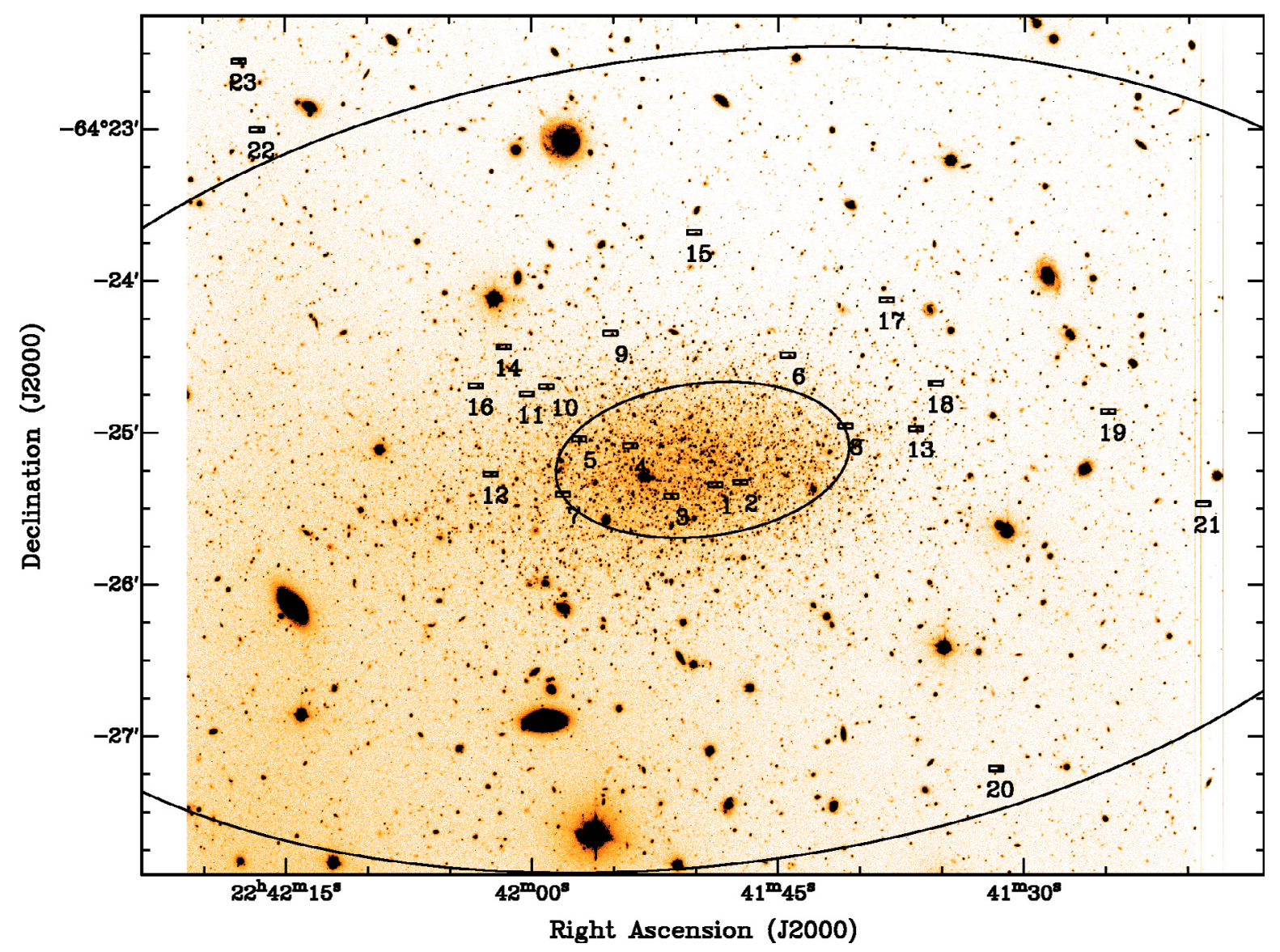

Fig. 1. Optical image ( $R$-band, FORS1) of Tucana overlaid with the positions of the slits covering the stars for which we obtained spectra with FORS2. The small and large ellipses show the locations and orientations of the geometric core and "tidal" radii. North is up and East to the left.

where the error ranges corresponding to $68 \%$ confidence intervals are derived from the relevant marginalised $1 \mathrm{D}$ distributions.

A straightforward statistical average of the same distribution of stars gives results very similar to the maximum likelihood fit since the velocity errors are generally much smaller than the apparent dispersion. We also note the apparent non-Gaussian nature of the distribution compared to the fit,

\subsection{Internal kinematics}

Tidal influences are unlikely to be significant at this isolated location. We examined the velocity distribution as a function of spatial position, and in particular searched for signs of possible rotational signatures. Tucana has a large ellipticity $e=0.48$ with a position angle running almost parallel to the RA axis (PA $97 \mathrm{deg}$, Mateo 1998). The spatial distribution of spectroscopic measurements provides reasonable coverage along this major axis but is somewhat more restricted along the minor axis (see Fig. 1). With this caveat, the most prominent correlation found was with respect to projected major axis position. Figure 6 illustrates this and shows the radial velocities of probable Tucana member stars as a function of their projected major axis distance. The dot-dash line shows a fiducial flat rotation curve of $15.0 \mathrm{~km} \mathrm{~s}^{-1}$ in the observed frame, and the solid line the fitted slope, $6.5 \times R / R_{\text {core }} \pm 2.9 \mathrm{~km} \mathrm{~s}^{-1}$, of a solid body rotation curve constrained to pass through the systemic velocity at the origin.

The reliabilty of our measurements and the accompanying errors were vigourously tested. This included independently analysing data taken for Tucana on different nights to compare
Table 2. Observational log.

\begin{tabular}{ccccc}
\hline \hline Date & $\begin{array}{c}\text { UT (end) } \\
\text { (h:m:s) }\end{array}$ & Airmass & $\begin{array}{c}\text { Seeing } \\
\left({ }^{\prime \prime}\right)\end{array}$ & $\begin{array}{c}\text { Dataset ID } \\
\text { (FORS2.2002-08-) }\end{array}$ \\
\hline $12 / 08 / 2002$ & $04: 09: 42$ & 1.383 & 0.98 & $12 \mathrm{~T} 04: 09: 42.883$ \\
& $04: 36: 32$ & 1.347 & 0.88 & $12 \mathrm{~T} 04: 36: 32.163$ \\
& $05: 11: 55$ & 1.316 & 0.71 & $12 \mathrm{~T} 05: 11: 55.313$ \\
& $05: 38: 44$ & 1.303 & 0.66 & $12 \mathrm{~T} 05: 38: 44.081$ \\
$13 / 08 / 2002$ & $03: 14: 54$ & 1.483 & 1.15 & $13 \mathrm{~T} 03: 14: 54.375$ \\
& $03: 41: 41$ & 1.425 & 1.03 & $13 \mathrm{~T} 03: 41: 41.247$ \\
$14 / 08 / 2002$ & $01: 37: 57$ & 1.822 & 1.03 & $14 \mathrm{~T} 01: 37: 57.100$ \\
& $02: 04: 44$ & 1.699 & - & $14 \mathrm{~T} 02: 04: 44.521$ \\
& $02: 32: 36$ & 1.594 & - & $14 \mathrm{~T} 02: 32: 36.985$ \\
& $02: 59: 27$ & 1.513 & 0.72 & $14 \mathrm{~T} 02: 59: 27.785$ \\
& $03: 30: 20$ & 1.439 & 0.77 & $14 \mathrm{~T} 03: 30: 20.610$ \\
& $03: 57: 08$ & 1.39 & 0.85 & $14 \mathrm{~T} 03: 57: 08.873$ \\
\hline
\end{tabular}

For each observation the exposure time is $1560 \mathrm{~s}$.

the results. We also tested that our method did not find a rotation signal due to the alignment of the CCDs on the sky, or our data analysis techniques by analysing data from the FORS2 archive for observations of fields in the Large Magellanic Cloud and in a number of globular clusters.

These apparent rotation signatures we find in Tucana are comparable with values recently found for other dSphs e.g. Cetus (Lewis et al. 2007) and Sculptor (Battaglia et al. 2008a). 

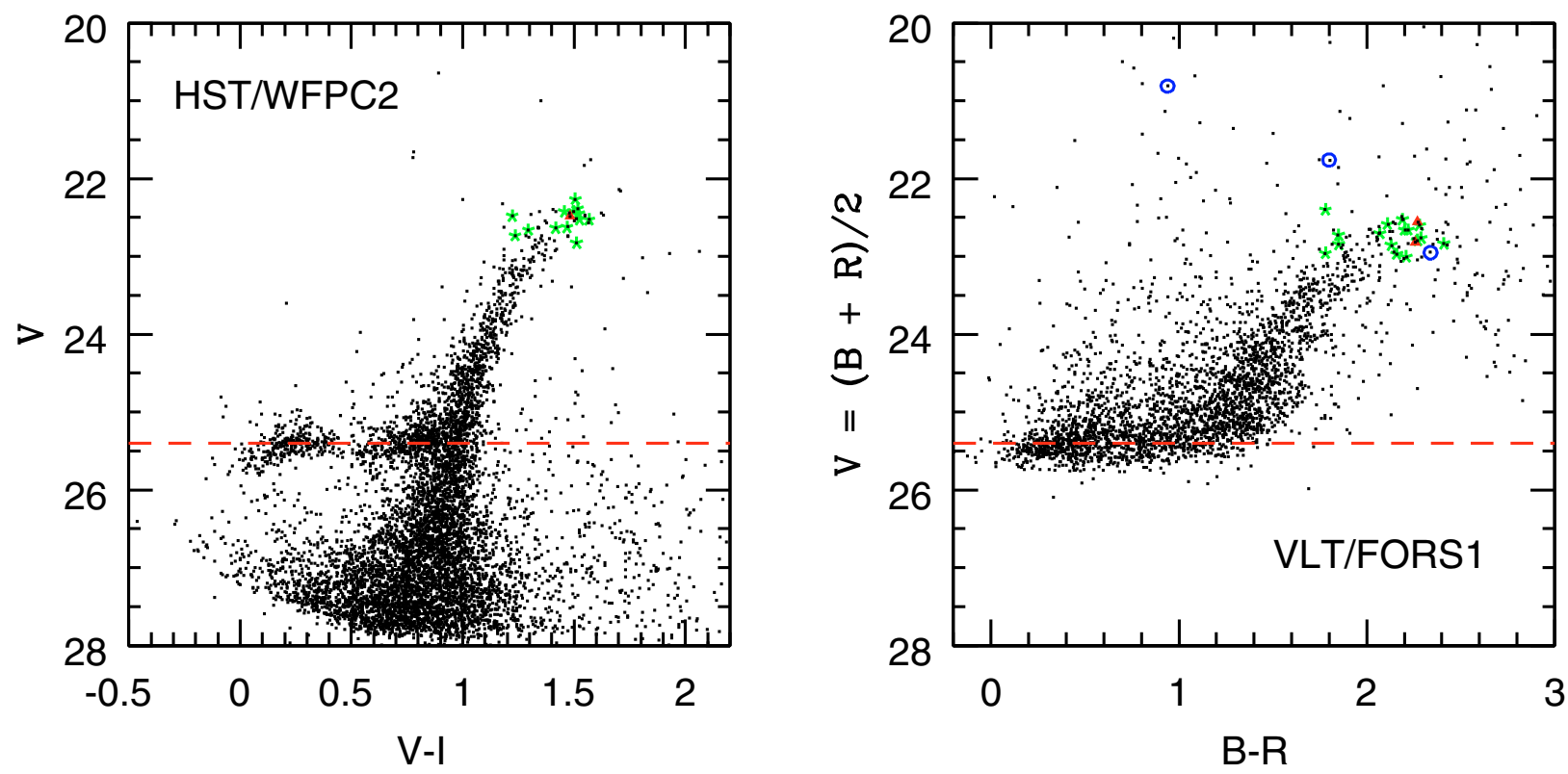

Fig. 2. Colour-magnitude diagrams of Tucana, the one on the left comes from archival HST/WFPC2 data (Holtzman et al. 2006) and the one on the right comes from UT1/FORS1 imaging (Tolstoy et al. 2000). In both CMDs the targets selected for spectroscopy are plotted as symbols different from the underlying CMD. In both cases likely velocity members are plotted as green star shaped symbols and non-members are plotted as blue open circles. The stars which are probably members but which we do not include in our analysis are plotted as red solid triangles. Also plotted on the CMDs is the position of $V_{\mathrm{HB}}=25.4$, determined visually from the HST CMD, and this is the value used in the determination of the Ca II triplet metallicities. Not all the stars have both HST and FORS photometry, see Table 3, which is why not all spectroscopic stars are plotted on both CMDs, one star does not appear on either CMD (\#21).

\subsection{Mass:to:Light ratio}

The photometric structural parameters of Tucana presented in Saviane et al. (1996) imply a total luminosity of $5.6 \pm 1.6 \times$ $10^{5} L_{\odot}$. Several different estimates of the central surface brightness, $\Sigma_{o}$, are given by Saviane et al. (1996) so for convenience we have used the King profile fit central surface brightness of $24.76 \pm 0.18 \mathrm{mag} / \operatorname{arcsec}^{2}$ as a fiducial value in this section. At a distance of $880 \mathrm{kpc}$ this translates to a central surface brightness of $4.3 \pm 0.8 L_{\odot} \mathrm{pc}^{-2}$, although we caution that the errors may be underestimated given the range of possible central surface brightness values.

Following Richstone \& Tremaine (1986) we can estimate the mass-to-light $(M / L)$ ratio by making the simplifying assumption that mass follows light in this system and then the total $M / L$ is given by

$\frac{M}{L}=\eta \frac{9 \sigma_{\mathrm{v}}^{2}}{2 \pi G \Sigma_{o} r_{\mathrm{h}}}$

where $\eta$ is a dimensionless constant of order unity for a wide variety of different light profiles. The appropriate half-light radius $r_{\mathrm{h}}=221 \mathrm{pc}$ (or 51") we define using the geometric King core, $R_{\text {core }}$, and tidal radii, $R_{\text {tidal }}$ listed in Table $1 . \sigma_{\mathrm{v}}$ is the central velocity dispersion, not including stars \#10, \#21 \& \#22. This gives a mass-to-light ratio of $M / L=105_{-49}^{+95}$, where the errors include a contribution from uncertainties in both the kinematics and the photometry. This implies a total mass of the system of $\approx 6 \times 10^{7} M_{\odot}$.

However, as noted in the previous section, there is a systematic trend in velocity as a function of position along the major axis, possibly due to inherent rotation. If we remove the "rotational" signature using a linear fit to the marginalised 1D maximum likelihood solutions the systemic velocity becomes $v_{\text {helio }}=+194.0 \pm 4.3 \mathrm{~km} \mathrm{~s}^{-1}\left(V_{\mathrm{GC}}=+98.9 \mathrm{~km} \mathrm{~s}^{-1}\right)$ and the velocity dispersion $\sigma_{v}=15.8_{-3.1}^{+4.1} \mathrm{~km} \mathrm{~s}^{-1}$. This implies a pressure supported mass of $\approx 5 \times 10^{7} M_{\odot}$. The rotational velocity is $\approx 16 \mathrm{~km} \mathrm{~s}^{-1}$ at a radius of $\approx 2 \times r_{\mathrm{h}}$, beyond which we have no statistics (see Sect. 3.1). However, we also note that for the King model, $87 \%$ of the light is enclosed within this radius and, if mass follows light in this system, this is also $87 \%$ of the mass. The equivalent rotationally supported mass within $\approx 2 \times r_{\mathrm{h}}$ is $\approx 3 \times 10^{7} M_{\odot}$, which is comparable to the pressure supported mass.

If we have detected a genuine signature of rotation, then it implies that the ratio of rotational support to pressure support in this system is of order unity. This value lies between what is typical for classical dSphs and dwarf irregular (dIrr) galaxies. It is notoriously hard to measure rotation in systems where it is of a similar order to the velocity dispersion. This means that not many dwarf systems have been studied with the required detail. These results for Tucana are broadly similar, although somewhat higher, to what has been found in Sculptor dSph (Battaglia et al. 2008a) and Cetus dSph (Lewis et al. 2007) which are similar luminosity (and mass) systems to Tucana, with similar star formation histories. As with Cetus and Sculptor the ellipticity of Tucana is broadly consistent with being flattened by rotation (Binney 1978). Looking at higher luminosity dwarf irregular galaxies the kinematic studies have been carried out exclusively with the H I gas. This means that the H I kinematics, specifically the velocity dispersion, cannot be directly compared to stellar velocity dispersion measures. Stellar kinematics are dominated by the mass of the galaxy and gas kinematics are most likely dominated by small scale interstellar medium processes, as witnessed by the fact that basically any galaxy from dIrr to large spirals, regardless of mass or rotation velocity, appears to have the same H I velocity dispersion $\approx 10 \mathrm{~km} \mathrm{~s}^{-1}$ (e.g., Mateo 1998; Lo et al. 1993). 
Table 3. Physical parameters of stars in our FORS2 field.

\begin{tabular}{|c|c|c|c|c|c|c|c|c|c|c|c|c|}
\hline N. & $\begin{array}{c}\text { RA } \\
(\mathrm{J} 2000)\end{array}$ & $\begin{array}{c}\text { Dec } \\
(\mathrm{J} 2000)\end{array}$ & $\begin{array}{c}V \\
(1)\end{array}$ & $\begin{array}{l}V-I \\
(1)\end{array}$ & $\begin{array}{l}R \\
(2)\end{array}$ & $\begin{array}{c}B-R \\
\text { (2) }\end{array}$ & $\begin{array}{c}r^{a} \\
(\operatorname{arcsec})\end{array}$ & $\begin{array}{c}S / N \\
\left(\AA^{-1}\right)\end{array}$ & $\begin{array}{c}V_{\text {hel }} \\
\left(\mathrm{km} \mathrm{s}^{-1}\right)\end{array}$ & Member? & $\begin{array}{c}\text { Eq. width } \\
(\AA)\end{array}$ & $\begin{array}{c}{[\mathrm{Fe} / \mathrm{H}]} \\
\operatorname{dex}\end{array}$ \\
\hline 1 & 224148.8 & -642520.4 & 22.48 & 1.22 & 21.80 & 1.85 & 10.8 & 16.1 & $172.0 \pm 9.4$ & $\mathrm{Y}$ & $4.11 \pm 0.28$ & -1.82 \\
\hline 2 & 224147.3 & -642519.5 & 22.53 & 1.57 & 21.62 & 2.29 & 17.1 & 23.2 & $184.6 \pm 5.7$ & Y & $4.61 \pm 0.20$ & -1.59 \\
\hline 3 & 224151.5 & -642525.0 & 22.64 & 1.42 & 21.89 & 2.16 & 18.7 & 22.1 & $200.7 \pm 6.4$ & $\mathrm{Y}$ & $3.66 \pm 0.22$ & -1.98 \\
\hline 4 & 224154.0 & -642505.1 & 22.39 & 1.52 & 21.56 & 2.20 & 29.1 & 30.5 & $180.0 \pm 5.5$ & $\mathrm{Y}$ & $3.46 \pm 0.14$ & -2.14 \\
\hline 5 & 224157.1 & -642502.4 & 22.82 & 1.51 & 21.79 & 2.13 & 49.3 & 19.5 & $187.5 \pm 6.7$ & $\mathrm{Y}$ & $3.84 \pm 0.23$ & -1.85 \\
\hline 6 & 224144.4 & -642429.2 & 22.42 & 1.46 & - & - & 53.7 & 18.8 & $159.6 \pm 10.0$ & $\mathrm{Y}$ & $2.94 \pm 0.36$ & -2.36 \\
\hline 7 & 224158.1 & -642524.3 & 22.66 & 1.30 & 21.92 & 1.85 & 56.6 & 15.6 & $225.2 \pm 6.9$ & Y & $4.16 \pm 0.29$ & -1.75 \\
\hline 8 & 224140.9 & -642457.2 & 22.62 & 1.47 & 21.90 & 2.21 & 58.0 & 14.5 & $205.7 \pm 12.5$ & $\mathrm{Y}$ & $4.99 \pm 0.41$ & -1.40 \\
\hline 9 & 224155.2 & -642420.6 & 22.42 & 1.50 & 21.53 & 2.11 & 62.1 & 28.6 & $204.8 \pm 8.4$ & Y & $3.43 \pm 0.15$ & -2.14 \\
\hline $10^{c}$ & 224159.1 & -642441.6 & 22.48 & 1.48 & 21.69 & 2.26 & 68.2 & 25.1 & $234.5 \pm 9.6$ & $\mathrm{Y}$ ? & $3.03 \pm 0.21$ & -2.30 \\
\hline 11 & 224200.3 & -642444.5 & 22.73 & 1.24 & 22.07 & 1.78 & 74.3 & 14.2 & $192.7 \pm 12.0$ & $\mathrm{Y}$ & $4.22 \pm 0.32$ & -1.71 \\
\hline 12 & 224202.5 & -642516.2 & 22.26 & 1.51 & 21.43 & 2.19 & 83.7 & 18.8 & $232.8 \pm 6.3$ & $\mathrm{Y}$ & $3.96 \pm 0.33$ & -1.95 \\
\hline 13 & 224136.6 & -642458.2 & 22.34 & 1.60 & 21.46 & 2.28 & 85.1 & 25.3 & $169.0 \pm 7.0$ & Y & $2.87 \pm 0.18$ & -2.41 \\
\hline 14 & 224201.7 & -642425.8 & - & - & 21.55 & 2.22 & 89.2 & 25.6 & $195.1 \pm 9.7$ & $\mathrm{Y}$ & $2.80 \pm 0.21$ & -2.39 \\
\hline 15 & 224150.1 & -642340.7 & - & - & 21.51 & 1.78 & 90.3 & 29.4 & $172.9 \pm 7.3$ & $\mathrm{Y}$ & $4.08 \pm 0.14$ & -1.86 \\
\hline 16 & 224203.4 & -642441.3 & - & - & 21.63 & 2.41 & 94.2 & 22.2 & $200.2 \pm 6.8$ & $\mathrm{Y}$ & $5.38 \pm 0.21$ & -1.17 \\
\hline 17 & 224138.4 & -642407.4 & 22.52 & 1.53 & 21.67 & 2.07 & 96.5 & 23.1 & $181.3 \pm 9.2$ & $\mathrm{Y}$ & $3.18 \pm 0.22$ & -2.22 \\
\hline 18 & 224135.4 & -642440.4 & 22.45 & 1.52 & 21.66 & 2.27 & 96.9 & 21.1 & $197.5 \pm 7.3$ & Y & $4.40 \pm 0.31$ & -1.70 \\
\hline 19 & 224124.9 & -642451.3 & - & - & 21.78 & 2.34 & 161.1 & 16.7 & $70.4 \pm 8.1$ & $\mathrm{~N}$ & $3.51 \pm 0.32$ & - \\
\hline 20 & 224131.7 & -642712.6 & - & - & 20.34 & 0.94 & 168.0 & 36.3 & $78.1 \pm 37.8$ & $\mathrm{~N}$ & $1.11 \pm 0.16$ & - \\
\hline 21 & 224119.1 & -642527.8 & - & - & - & - & 200.2 & 18.1 & $234.9 \pm 6.3$ & Y? & $3.88 \pm 0.37$ & $-1.92^{d}$ \\
\hline 22 & 224216.7 & -642259.9 & - & - & 21.42 & 2.27 & 219.2 & 26.8 & $201.6 \pm 11.5$ & Y? & $3.53 \pm 0.20$ & -2.06 \\
\hline 23 & 224217.8 & -642232.8 & - & - & 20.86 & 1.80 & 241.7 & 19.0 & $5.6 \pm 5.6$ & $\mathrm{~N}$ & $4.19 \pm 0.29$ & - \\
\hline
\end{tabular}

(1) From WFPC2 photometry, (Holtzman et al. 2006); (2) from FORS1 photometry, (Tolstoy et al. 2000). ${ }^{a}$ Projected distance from the centre of Tucana; ${ }^{b}$ sum of the two strongest Ca II lines; ${ }^{c}$ double star; ${ }^{d}$ Using the fiducial value of $V=22.5$, coming from the signal-to-noise $(S / N)$ of the star.

\subsection{Metallicity}

We have estimated the metallicities of the observed stars in Tucana using the well-studied correlation between the equivalent widths $(E W)$ of $\mathrm{Ca}$ II lines and $[\mathrm{Fe} / \mathrm{H}]$ abundance, first outlined by Armandroff \& Da Costa (1991). The validity of this approach over the range $-2.5<[\mathrm{Fe} / \mathrm{H}]<-0.5$ has been firmly established through the comparison of Ca II $E W$ s with high resolution metallicities for a large number of stars in globular clusters (Rutledge et al. 1997) and in dSph (Battaglia et al. 2008b). We used the preferred calibration determined by Battaglia et al. according to which the $[\mathrm{Fe} / \mathrm{H}]$ abundance is given by:

$[\mathrm{Fe} / \mathrm{H}]=-2.81+0.44\left(E W_{8542}+E W_{8662}+0.64\left(V-V_{\mathrm{HB}}\right)\right)$

where $E W_{\lambda}$ is the equivalent width of the line at wavelength $\lambda$ (the two brightest Ca II lines), $V$ and $V_{\mathrm{HB}}$ are the $V$-band apparent magnitudes of the star and of the horizontal branch $\left(V_{\mathrm{HB}}=25.4\right)$ of the system respectively, see also (Cole et al. 2004). In cases where no $V$-band data were available we used the conversion $V=(B+R) / 2$.

Figure 7 shows the histogram distribution of the metallicities. The solid curve is a maximum likelihood fit of a Gaussian function normalised and overlaid on a histogram of all 20 potential Tucana members ${ }^{1}$. The likelihood fit indicates a mean metallicity of $[\mathrm{Fe} / \mathrm{H}]=-1.95 \pm 0.15$ and a dispersion of $\sigma=$ $0.32 \pm 0.06$ dex. Similar, although slightly higher, values for the average metallicity have been found by Saviane et al. (1996) and Castellani et al. (1996) from comparing the CMD of Tucana with those of globular clusters. The relatively large spread in metallicity that we found is partially due to the errors in the determination of the equivalent widths and partially to a genuine intrinsic

\footnotetext{
1 We used a fiducial value of $V=22.5$ for the star with no photometry since the signal-to-noise in the spectrum is comparable on average with stars of this magnitude.
}

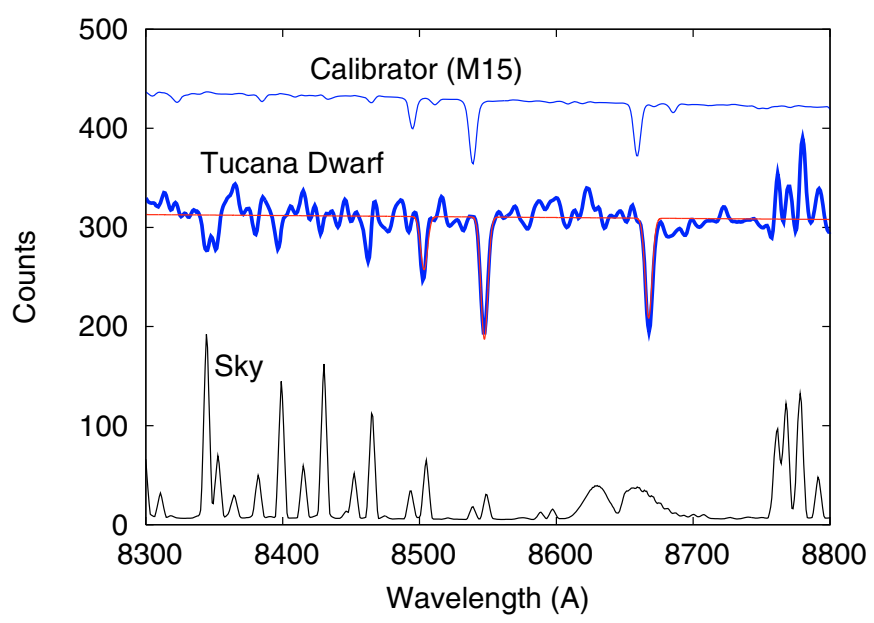

Fig. 3. A sample spectrum (thick blue line) of a star in Tucana (\#4 in Table 3). The thin (red) line overlaid shows our fit of the Ca II triplet absorption lines using Gaussian profiles. The upper spectrum (thin blue line) belongs to a star in the calibration globular cluster M 15. The bottom spectrum shows the sky emission spectrum scaled down by a factor 30. Note the clear shift between the location of the Ca II triplet in M 15 and Tucana.

dispersion in metallicity. From Table 3 it can be seen that the typical error in $E W$ is $\approx 0.25 \AA$ which translates into an error in metallicity of $\approx 0.1$ dex. Calibration errors of $\approx 0.1$ dex tend to affect all stars equally over this sort of metallicity range (Battaglia et al. 2008b). This suggests that most of the observed metallicity spread is intrinsic to Tucana and that an extended period of star formation, albeit $\approx 10$ Gyrs ago, is the most likely explanation.

There is no evidence in our spectroscopic sample for a metallicity gradient in Tucana, as has been seen in the variable 


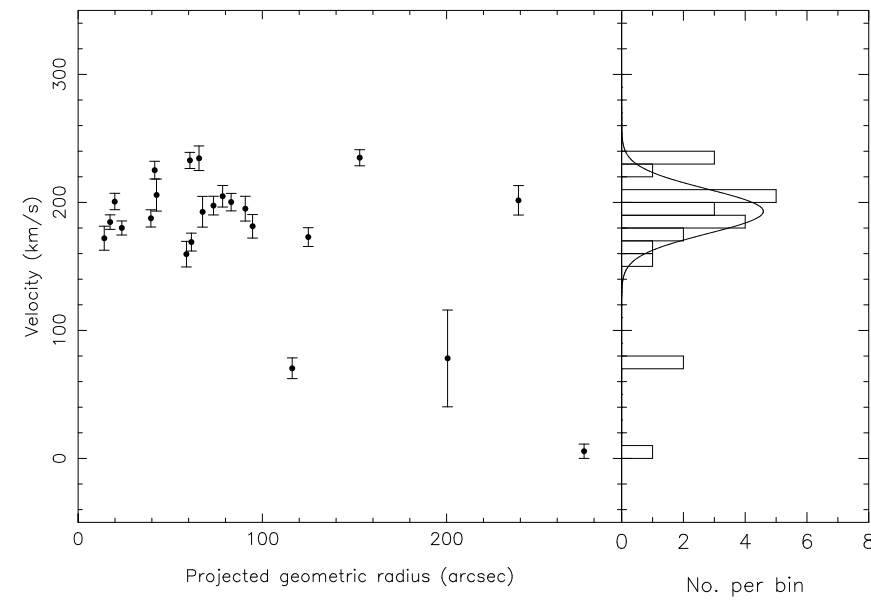

Fig. 4. Left panel: the projected geometric elliptical radial distribution of the velocities for all the stars in Table 3. Note the clear divisions between central likely members, outer possible members and lower velocity foreground stars. Right panel: a histogram of the velocity distribution of the most probable 17 members sample, with the maximum likelihood Gaussian model fit, $v_{\text {hel }}=+193.0 \pm 4.9 \mathrm{~km} \mathrm{~s}^{-1}$ and $\sigma_{v}=17.4 \mathrm{~km} \mathrm{~s}^{-1}$, overlaid for reference.
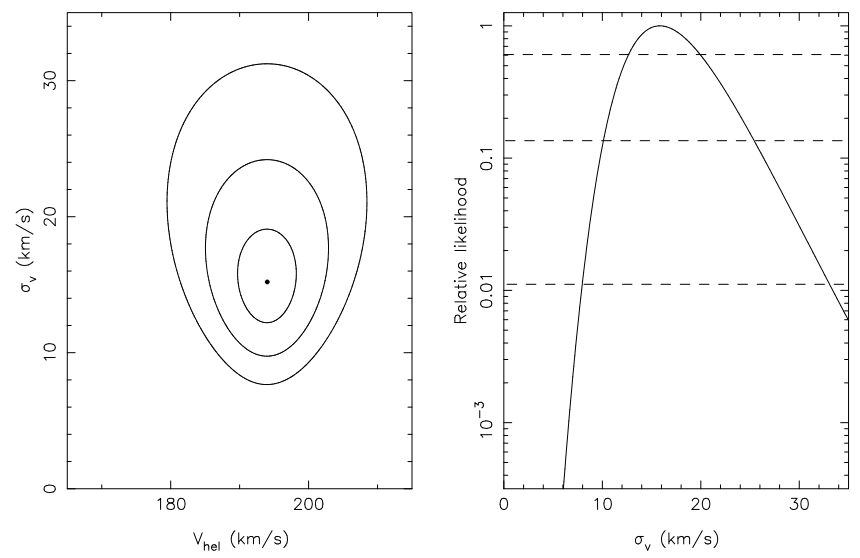

Fig. 5. Left panel: the 2D maximum likelihood solution for the 17 members of Tucana lying within a projected geometric radius of 125 arcsec, contours are at the $1,2 \& 3 \sigma$ levels. The right panel shows the marginalised (with respect to systemic velocity) "central" velocity dispersion solution, with dashed lines denoting the $1,2 \& 3 \sigma$ confidence levels.

stars (e.g., Bernard et al. 2008), or in spectroscopic studies of other dSph galaxies (e.g., Sculptor: Tolstoy et al. 2004; Fornax: Battaglia et al. 2006).

\section{Discussion}

One of the obvious features that distinguishes dSph galaxies from other dwarf galaxies, such as dIrrs, is the lack of gas and ongoing star formation. The region of sky around Tucana has been observed at 21-cm wavelength by Oosterloo et al. (1996). They discovered an $\mathrm{HI}$ cloud at a projected distance of $15^{\prime}$ to the north-east of Tucana with a weighted mean heliocentric velocity of $\approx 130 \mathrm{~km} \mathrm{~s}^{-1}$. Although the cloud has a head-tail shape pointing at Tucana, Oosterloo et al. considered the association with Tucana not to be very probable. On the other hand, Blitz \& Robishaw (2000) reviewed H I detections which are possibly associated with dSph galaxies and described Tucana as a probable

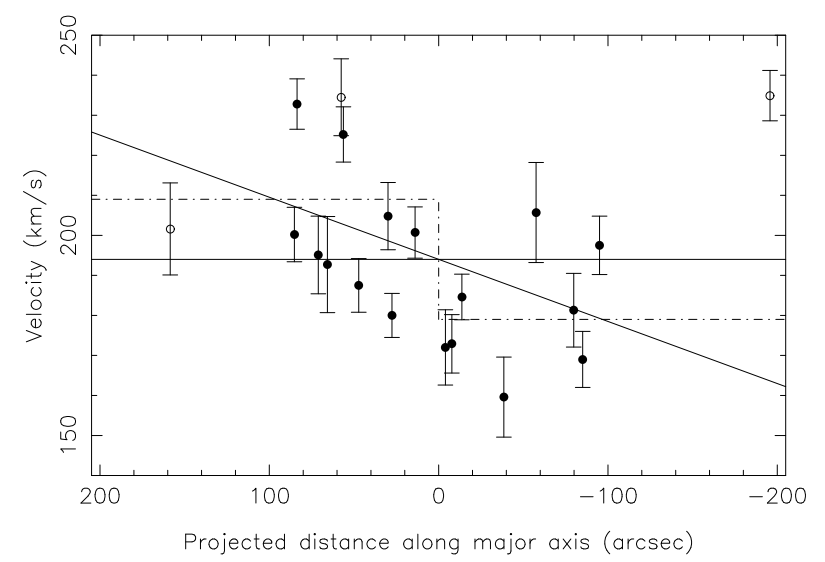

Fig. 6. Heliocentric radial velocities of the 20 likely member stars in Tucana as a function of their projected major axis distance (East is to the left). The three open circles are the likely binary star (\#10), the star lacking photometry (\#21), and the star beyond the tidal radius (\#22). The solid line shows a solid body rotation curve with slope $6.5 \times R / R_{\text {core }} \pm 2.9 \mathrm{~km} \mathrm{~s}^{-1}$ fitted to the filled circle points. The dotdashed line shows a fiducial flat rotation curve with a rotation velocity of $15.0 \mathrm{~km} \mathrm{~s}^{-1}$.

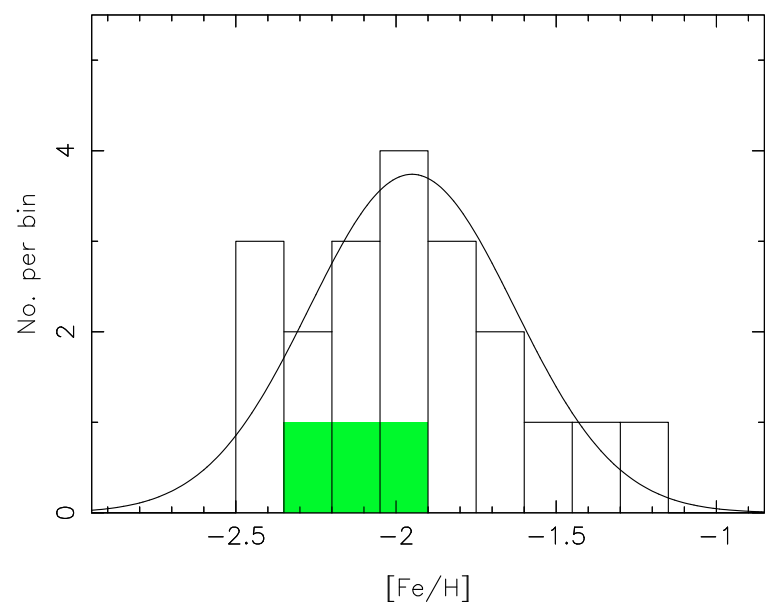

Fig. 7. Distribution of metallicities for the 20 likely member stars in Tucana estimated from Ca II triplet fitting. The 3 stars which were removed from the kinematic analysis are shown shaded. The solid curve shows an overlaid maximum likelihood fit of a Gaussian model with a mean metallicity of $[\mathrm{Fe} / \mathrm{H}]=-1.95 \pm 0.15 \mathrm{dex}$ and dispersion (sigma) of $0.32 \pm 0.06$ dex.

H I detection as do Bouchard et al. (2006) using new observations with the Parkes telescope.

With our determination of the systemic velocity of Tucana we can firmly exclude an association between H I and Tucana. From the channel maps, published by Oosterloo et al., the H I emission of the cloud has already completely disappeared at a heliocentric velocity of $165 \mathrm{~km} \mathrm{~s}^{-1}$, which is $30 \mathrm{~km} \mathrm{~s}^{-1}$ away from the velocity of Tucana. Most probably, the H I cloud is associated with the Magellanic Stream. In Fig. 8 we show two H I channel maps, obtained from the Leiden-Argentina-Bonn (LAB) H I survey of the Milky Way (Kalberla et al. 2005). The top panel shows an integration of $40 \mathrm{~km} \mathrm{~s}^{-1}$ around the heliocentric velocity of $130 \mathrm{~km} \mathrm{~s}^{-1}$, which is the velocity of the Oosterloo et al.'s cloud (the cross shows the position of Tucana). The cloud (which is not visible in Fig. 8) lies at the edge the H I emission coming from the Magellanic Stream. At our newly determined helio-centric optical velocity of Tucana of $\approx 190 \mathrm{~km} \mathrm{~s}^{-1}$ (bottom 


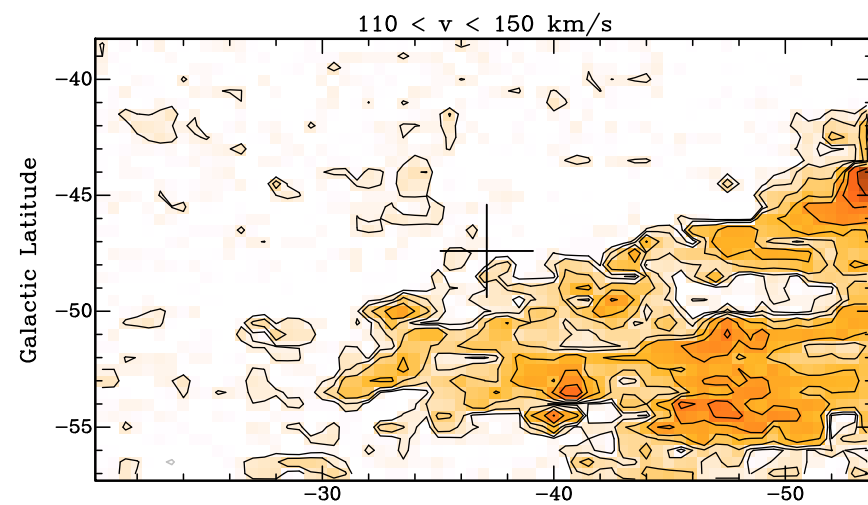

Galactic Longitude

$170<\mathrm{v}<210 \mathrm{~km} / \mathrm{s}$

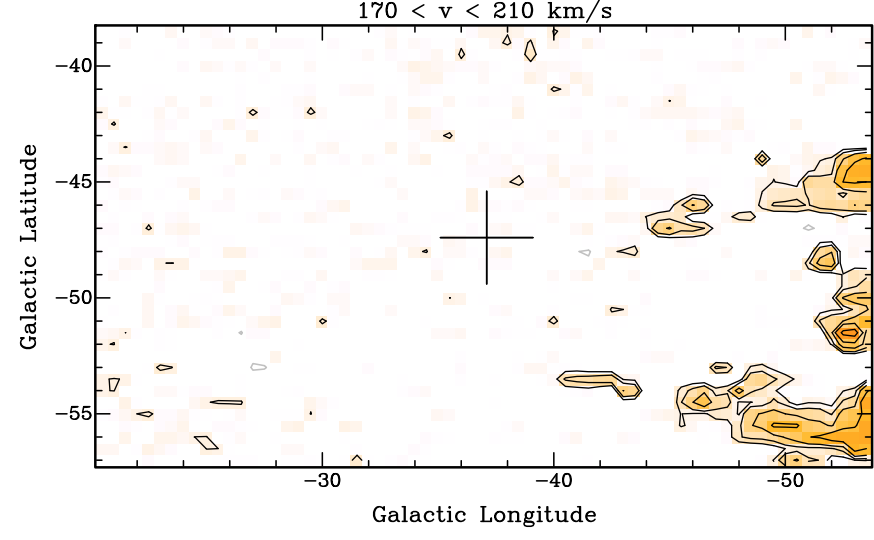

Fig. 8. Neutral hydrogen emission in the field around Tucana (cross) from the LAB H I survey. The two maps are integrated over $40 \mathrm{~km} \mathrm{~s}^{-1}$ around two different l.o.s. velocities: $130 \mathrm{~km} \mathrm{~s}^{-1}$ (top panel) and $190 \mathrm{~km} \mathrm{~s}^{-1}$ (bottom panel). Most of the emission visible here comes from the Magellanic Stream; the SMC is at $b=-57.2, l=-44.3$, just outside the frames. Contours levels: $-2.5,2.5,5,10,20,40,80,160$ in units of $\sigma$.

panel) the foreground emission has totally disappeared and if there were H I associated with Tucana, it would be clearly separated from the local emission. The more sensitive observations of Oosterloo et al. did not detect any emission at these velocities so we can conclude that Tucana has no neutral hydrogen associated with it down to their detection limit of $1.5 \times 10^{4} M_{\odot}$ $(5 \sigma)$.

The lack of neutral hydrogen is consistent with the lack of recent star formation in Tucana. A key question is how did Tucana lose its gas? The two main possibilities are tidal/ram-pressure interactions with large galaxies (e.g., Mayer et al. 2001) or gas blow-out from stellar feedback (e.g., Mac Low \& Ferrara 1999). The proximity of most of the dSphs to the large members of the Local Group shows that the former mechanism must play an important role. However, given that Tucana is at a large distance from the Milky Way and even further from M31, one would not expect interaction to play a role in removing its gas. For example, one of Tucana's closest neighbours is the Phoenix dwarf galaxy, at a separation of $560 \mathrm{kpc}$, but along a similar line of sight although with a very different radial velocity, at $-13 \pm 9 \mathrm{~km} \mathrm{~s}^{-1}$ (Irwin \& Tolstoy 2002). Phoenix is generally considered to be a transition-type object, in the process of losing its gas (Young et al. 2007).

The heliocentric velocity, $v_{\text {helio }}=+194.0 \mathrm{~km} \mathrm{~s}^{-1}$, of Tucana corresponds to a velocity with respect to the barycentre of the Local Group of $v_{\text {LGSR }}=+73.3 \mathrm{~km} \mathrm{~s}^{-1}$ (taking the mass ratio of M 31:Milky Way as 2:1). Thus, given the timescales and distance involved, Tucana has not reached its apocentre (if bound) and it is still moving away from the Local Group barycentre faster than most other galaxies at similar distances (e.g., SagDig and Aquarius). In Fig. 9 we show the Local-Group-centric velocity of dwarf galaxies versus their distance from the Local Group barycentre (updated from Lewis et al. 2007). Tucana falls in the region believed to be associated with the bridge of nearby galaxies toward the Sculptor Group (on the right side of the plot). Given that the Sculptor Group is only 30 degrees away from Tucana, it is quite possible that Tucana belongs to a bridge (or a cosmological filament?) connecting the Sculptor group with the Local Group.

Another possibility arises from interpreting the residual velocity of Tucana as a receding speed from the Local Group or from the Milky Way. In this scheme Tucana may be in a very elongated orbit but still bound to the Local Group. If we interpret its velocity as receding from the Milky Way $\left(v_{\mathrm{GSR}}=\right.$ $+98.9 \mathrm{~km} \mathrm{~s}^{-1}$ ), we can in principle extrapolate back to an encounter with our Galaxy at a $t_{\mathrm{enc}} \lesssim 10$ Gyrs ago. Interestingly, this is consistent with the epoch of the last burst of star formation in Tucana (Saviane et al. 1996). A fly-by Tucana-Milky-Way could have removed the remaining gas from the dwarf galaxy preventing further star formation.

It has also been shown from simulations that three-body interactions between dwarf galaxy satellites falling in towards a parent galaxy can result in the ejection of the smaller dwarf on a nearly radial orbit at high velocity (Sales et al. 2007). These simulations show that a dwarf galaxy ejected at $300 \mathrm{~km} \mathrm{~s}^{-1}$ from a pericentre approach of about $60 \mathrm{kpc}$, is still travelling outwards 7-8 Gyr later, at nearly $1 \mathrm{Mpc}$ away from the primary. This kind of ejection process might have resulted in the gas and stars parting ways, and a highly elliptical system. This is broadly consistent with what we observe for Tucana. A second effect of this ejection may be the presence of streaming motions (see also Mateo et al. 2008). The velocity gradient observed in Tucana, can in principle be the signature of streaming instead of rotation. However, given its position and velocity, Tucana must move almost radially with respect to the Local Group and the Milky Way which suggests any tidal distortion, or debris, should be closely aligned along the line-of-sight. It therefore seems unlikely for a tidal disturbance to be visible along the major axis perpendicular to the likely direction of motion.

\section{Conclusions}

With VLT/FORS2 we have studied the properties of 20 individual stars which are probable velocity members of the Tucana Dwarf Galaxy, and we have found that:

i) Tucana has a receding systemic velocity with respect to the Sun $\left(v_{\text {hel }}=+194.0 \pm 4.3 \mathrm{~km} \mathrm{~s}^{-1}\right)$, our Galaxy $\left(v_{\mathrm{GSR}}=\right.$ $+98.9 \mathrm{~km} \mathrm{~s}^{-1}$ and the Local Group $v_{\text {LGSR }}=+73.3 \mathrm{~km} \mathrm{~s}^{-1}$. Its velocity dispersion is $17.4 \mathrm{~km} \mathrm{~s}^{-1}$ uncorrected for possible rotation; falling to $15.4 \mathrm{~km} \mathrm{~s}^{-1}$ after removing a linear rotation signature.

ii) Tucana has no cold gas associated, the upper limit for its gas content coming from Oosterloo et al. (1996) is $1.5 \times 10^{4} M_{\odot}$.

iii) All the stars that we observed have low metallicity with a mean value of $[\mathrm{Fe} / \mathrm{H}]=-1.95$ and a dispersion of $0.32 \mathrm{dex}$.

iv) There is evidence of regular rotation around the photometric minor axis with a velocity up to $v_{\text {rot }} \approx 16 \mathrm{~km} \mathrm{~s}^{-1}$ at $\approx 2 \times r_{\mathrm{h}}$.

In short, Tucana has physical properties similar to the other dwarf spheroidals in the Local Group but a peculiar location and peculiar receding velocity. It may be in a very elongated orbit 
LOCAL GROUP ENVIRONS

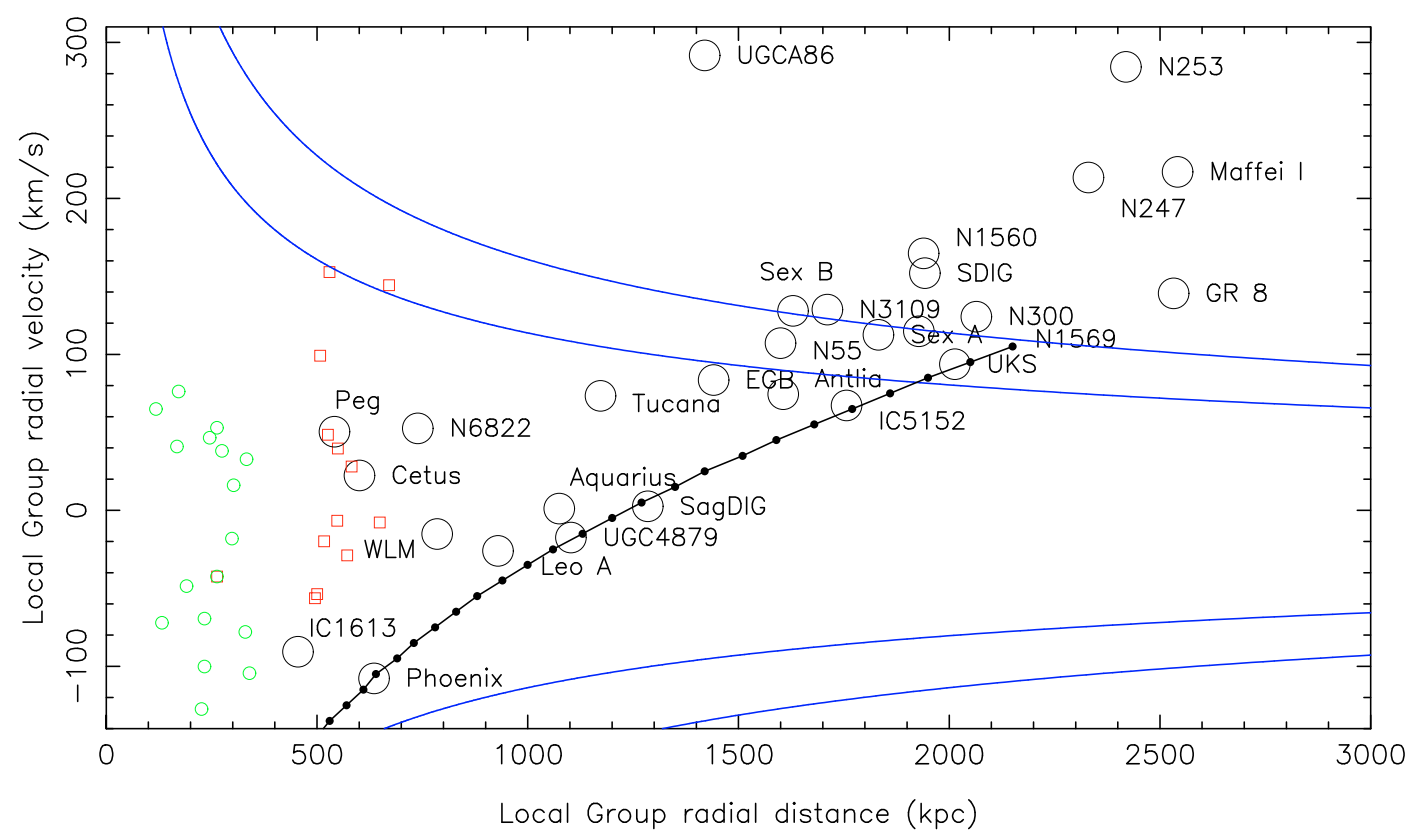

Fig. 9. The barycentric Local Group distance versus Local Group radial velocity for various nearby galaxies in the local universe, including the new data for Tucana. The upper and lower tracks are virial boundaries for purely radial motion and Local Group masses of $3.0 \times 10^{12} M_{\odot}$ and $4.5 \times 10^{12} M_{\odot}$ respectively. The "knotted" line denotes the limiting distance attainable for an object with purely radial motion in a notional 13 Gyrs since the Local Group origin. Tucana lies close to a bridge of galaxies leading to the Sculptor group, both in this projection and also spatially in the Local Group+Sculptor Group member galaxy distributions.

with respect to the Local Group or the Milky Way in which case it could have lost its gas (and stopped forming stars) at its passage through pericentre close to the Milky Way, $\approx 10$ Gyr ago. Another possibility is that it resides in a region bridging the Local Group and the Sculptor Group.

Acknowledgements. We thank Gianni Marconi and Thomas Szeifert for their help with the observations.

\section{References}

Armandroff, T. E., \& Da Costa, G. S. 1991, AJ, 101, 1329

Battaglia, G., Tolstoy, E., Helmi, A., et al. 2006, A\&A, 459, 423

Battaglia, G., Helmi, A., Tolstoy, E., et al. 2008a, ApJ, 681, L13

Battaglia, G., Irwin, M., Tolstoy, E., et al. 2008b, MNRAS, 383, 183

Bernard, E. J., Gallart, C., Monelli, M., et al. 2008, ApJ, 678, L21

Binney, J. 1978, MNRAS, 183, 501

Blitz, L., \& Robishaw, T. 2000, ApJ, 541, 675

Bouchard, A., Carignan, C., \& Staveley-Smith, L. 2006, AJ, 131, 2913

Castellani, M., Marconi, G., \& Buonanno, R. 1996, A\&A, 310, 715

Cole, A. A., Smecker-Hane, T. A., Tolstoy, E., Bosler, T. L., \& Gallagher, J. S. 2004, MNRAS, 347, 367

Holtzman, J. A., Afonso, C., \& Dolphin A. 2006, ApJS, 166, 534
Irwin, M., \& Tolstoy, E. 2002, MNRAS, 336, 643

Kalberla, P. M. W., Burton, W. B., Hartmann, D., et al. 2005, A\&A, 440, 775

Lavery, R. J., \& Mighell, K. J. 1992, AJ, 103, 81

Lewis, G. F., Ibata, R. A., Chapman, S. C., et al. 2007, MNRAS, 375, 1364

Lo, K. Y., Sargent, W. L. W., \& Young, K. 1993, AJ, 106, 507

Oosterloo, T., Da Costa, G. S., \& Staveley-Smith, L. 1996, AJ, 112, 1969

Mac Low, M.-M., \& Ferrara, A. 1999, ApJ, 513, 142

Mateo M. 1998, ARA\&A, 36, 435

Mateo, M., Olszewski, E. W., \& Walker, M. G. 2008, ApJ, 675, 201

Mayer, L, Governato, F., Colpi, M., et al. 2001, ApJ, 547, 123

McConnachie, A. W., Huxor, A., Martin, N. F., et al. 2008, ApJ, 688, 1009

Putman, M. E., Staveley-Smith, L., Freeman, K. C., Gibson, B. K., \& Barnes, D. G. 2003, ApJ, 586, 170

Richstone, D. O., \& Tremaine, S. 1986, AJ, 92, 72

Rutledge, G. A., Hesser, J. E., \& Stetson, P. B. 1997, PASP, 109, 907

Sales, L. V., Navarro, J. F., Abadi, M. G., \& Steinmetz, M. 2007, MNRAS, 379, 1475

Saviane, I., Held, E. V., \& Piotto, G. 1996, A\&A, 315, 40

Tolstoy, E., \& Irwin, M. 2000, MNRAS, 318, 1241

Tolstoy, E., Gallagher, J., Greggio, L., et al. 2000, ESO Messenger, 99, 16

Tolstoy, E., Irwin, M. J., Helmi, A., et al. 2004, ApJ, 617, L119

Whiting, A. B., Hau, G. K. T., \& Irwin, M. 1999, AJ, 118, 2767

Young, L. M. 2000, AJ, 119, 188

Young, L. M., Skillman, E. D., Weisz, D. R., \& Dolphin, A. E. 2007, ApJ, 659, 331 